\title{
Context-Driven Decision Support in Flexible Networked Organisations
}

\author{
Alexander Smirnov, Tatiana Levashova, and Nikolay Shilov \\ St. Petersburg Institute for Informatics and Automation \\ of the Russian Academy of Sciences, \\ 39, 14-th Line, 199178, St.-Petersburg, Russia \\ \{smir, oleg, nick\}iias.spb.su
}

\begin{abstract}
The paper proposes a context-driven approach to decision making in flexible networked organisations for the purpose of selecting partners for a specific task. The main idea of the approach is to represent the members of the networked organisation by services they provide. This makes it possible to replace the configuration of the networked organisation with that of services constituting it. Such representation benefits from the principles of the serviceoriented architectures, such as service autonomy, abstraction, standardisation, and reusability. For the interoperability purposes the services communicate in terms of a common ontology. To improve the flexibility of the operational decision support each particular situation is described by a narrow context that contains only information that is relevant to the situation.
\end{abstract}

Keywords: Operational decision support, service network, ontology, context.

\section{Introduction}

Global climate changes and globalization of the world economy set new goals for the manufacturing companies. One of such new requirements is sustainability. There are several definitions for this term (e.g., [1]) but all of them refer to being environment friendly and as a result aiming at long-term competitive advantage. One of such definitions is given in [2]: sustainable manufacturing is developing technologies to transform materials without emission of greenhouse gases, use of non-renewable or toxic materials or generation of waste.

The author of [2] also identifies five major means helping to make manufacturing more sustainable. These means include:

1. Use less material and energy.

2. Substitute input materials: non-toxic for toxic, renewable for non-renewable.

3. Reduce unwanted outputs: Cleaner production, Industrial symbiosis.

4. Convert outputs to inputs: recycling and all its variants.

5. Changed structures of ownership and production: product service systems, supply chain structure.

The first four means require technological advances and fall beyond the scope of this paper. Instead, the paper concentrates on structural changes in production. 
A context-driven service-oriented approach to decision support in flexible networked organisations is proposed for the purpose of selecting partners for a specific task. The approach also relies at profiling, which is important for determining members capable of carrying out a specified task with the given level of sustainability.

The paper is structured as follows. The context-driven service-oriented architecture is described in sec. 2. The application of the profiling technology is described in sec. 3. Major results are summarised in conclusions.

\section{Approach}

This work is a development of the idea presented in [3] aimed at knowledge management in production networks. The main idea of the developed approach is to represent the members of the flexible networked organisation by services they provide (Fig. 1). This would make it possible to replace the modelling of the networked organisation with that of services constituting it. The flexibility assumes efficient management of information and taking into account the dynamic environment. For this purpose the approach proposed actualises information in accordance with the current situation. An ontological model described by the Application Ontology (AO) is used in the approach to solve the problem of service heterogeneity. This model makes it possible to enable interoperability between heterogeneous information services due to provision of their common semantics [4]. Depending on the considered problem the relevant

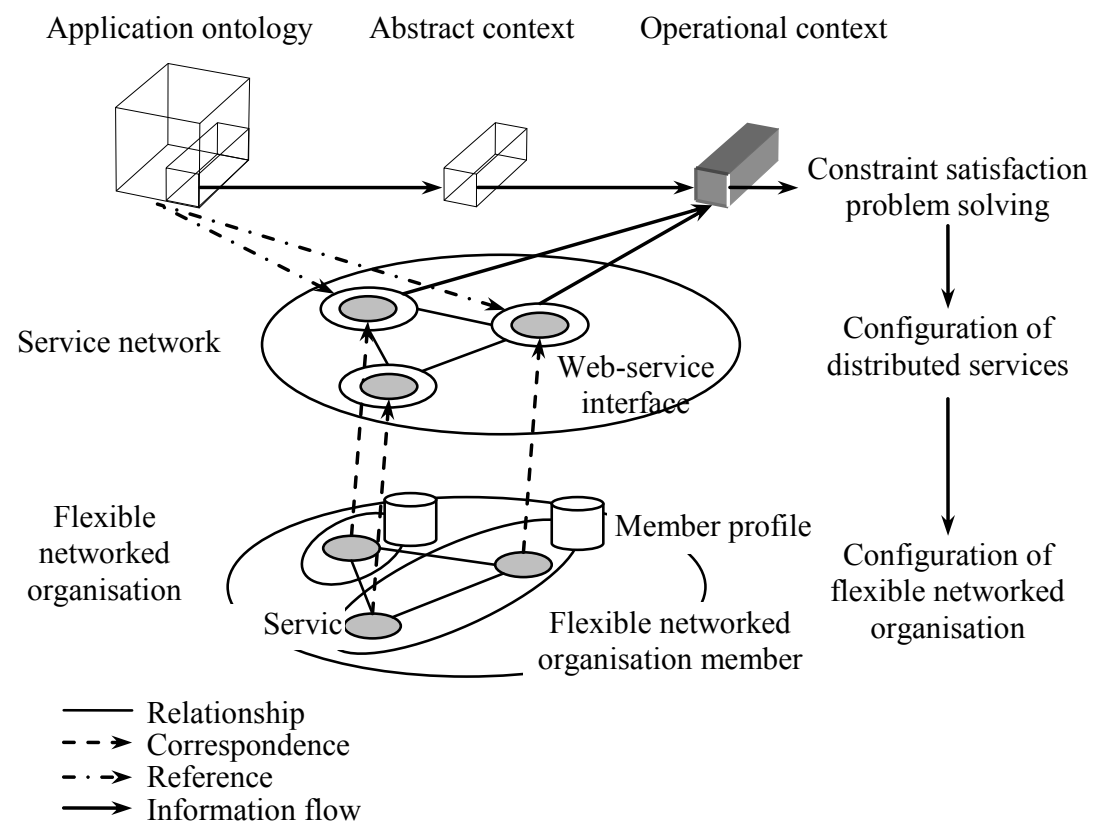

Fig. 1. Generic scheme of the approach 
part of the application ontology is selected forming the abstract context that, in turn, is filled with values from the sources resulting in the operational context. Application of the context model makes it possible to reduce the amount of information to be processed. This model enables management of information relevant for the current situation [5]. The operational context represents the constraint satisfaction problem that is used during configuration of services for problem solving. The access to the services, information acquisition, transfer, and processing (including integration) are performed via usage of the technology of Web-services.

The service-oriented architecture has a number of advantages resulting from its principles [6]. Among these the following once should be mentioned (the specifics related to flexible supply network modelling are indicated with italic):

1. Service Autonomy. Services engineered for autonomy exercise a high degree of control over their underlying run-time execution environment. Autonomy, in this context, represents the level of independence which a service can exert over its functional logic. With regard to networked organisations the autonomy also reflects independence of the network members, which in real life are independent companies.

2. Service Abstraction. Further supporting service autonomy, service-oriented architecture advocates that the scope and content of a service's interface be both explicitly described and limited to that which is absolutely necessary for the service to be effectively employed. Beyond the service interface, abstraction applies to any information, in any form, describing aspects of the service's design, implementation, employed technologies, etc. This principle helps to abstract from real services provided by the networked organisation members and concentrates on their modelling via Web-services.

3. Service Standardisation. As services are typically distributed throughout networks, they must be easily accessible by other entities in terms of discoverability and consequential invocation. Given this requirement, service-oriented architecture recommends that services adhere to standards, including, for example, standards for the language used to describe a service to prospective consumers. In the proposed approach the standardisation is achieved via usage of the common standards such as WSDL and SOAP as well as common terminology described by AO. As a result the services constituting the network are fully interoperable and can communicate with each other without any problems.

4. Service Reusability. Reusability is a central property of any successful service. It denotes the capacity of a service to be employed in support of not just one but rather a variety of business models. Service-oriented architecture promotes such functional reuse through stipulations for service autonomy and interface abstraction. With these features, the same service can be invoked by multiple consumers, operating in various business domains, without requiring the service provider to recode service internals for each application domain. Service reusability significantly facilitates the modelling process and decreases the amount of work required for model building. Besides, the existing services of flexible supply network members can be used.

In [6] two more service-oriented architecture principles are defined, namely service composability and service discovery. However, they are out of the scope of the presented approach. 


\section{Service-Oriented Architecture}

In the proposed service-oriented architecture (Fig. 2) two types of Web-services are distinguished: core Web-services and operational Web-services.

The core Web-services are intended to create the abstract context and to monitor the business environment. The core Web-services comprise:

- MonitoringService monitors the environment, identifies the types of problems arising, and produces corresponding messages.

- ManagementService manages Web-services to create the abstract context. It operates with the service registry where the core services are registered.

- AOAccessService provides access to the AO;

- AbstractContextService creates, stores, and reuses abstract contexts;

The operational Web-services organize a service network. To make the Web-services active components capable to negotiate an agent-based service model is used. Agents are intended to negotiate services' needs and possibilities in terms of the AO and "activate" Web-services when required. The services' needs and possibilities are respectively input and output arguments of the functions that the Web-services implement. The set of operational Web-services comprises:

- InformationSourceService - a set of Web-services responsible for interactions with information sources of different types and for processing information provided by these sources. The following main types of information sources are distinguished: sensors, databases, Web-sites, and humans.

- ProblemSolvingService - a set of Web-services responsible for problem solving.

- MemberProfileService creates, modifies, and updates profiles of the acting resources or, in other words, the members of the networked organisation; provides

\begin{tabular}{|c|c|c|}
\hline \multirow{4}{*}{$\begin{array}{c}\text { Core } \\
\text { Web-Services }\end{array}$} & MonitoringService & Problem type identification \\
\hline & ManagementService & Web-service registry \\
\hline & AOAccessService & Application ontology \\
\hline & AbstractContextService & Abstract context \\
\hline \multirow{3}{*}{$\begin{array}{c}\text { Operational } \\
\text { Web-Services }\end{array}$} & InformationSourceService & 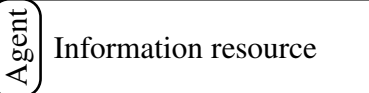 \\
\hline & ProblemSolvingService & 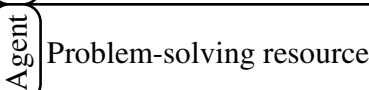 \\
\hline & $\begin{array}{c}\text { MemberProfileService } \\
\text { MemberInteractionService }\end{array}$ & Acting resource \\
\hline \multicolumn{2}{|c|}{ Self-organising Web-service network } & Operational context \\
\hline
\end{tabular}

Fig. 2. Service-oriented architecture 
access to these profiles; collects information about the members; in a context-based way accumulates information about the member activities; reveals preferences of the members.

- MemberInteractionService - a set of Web-services responsible for support of and interactions with the members. They communicate between the system and the members (i) providing system messages, context-sensitive help, pictures of the current situation, results of problem solving to the members, and (ii) delivering information from the members to the system.

\section{Profiling in Flexible Networked Organisation}

Choosing the right partner in sustainable production network is essential and should be based on the potential partners' competences and sustainability. For this purpose application of the profiling technology is proposed. The structure of the networked organisation member profile is given in Fig. 3. Member competence is determined by capabilities, capacities, price-list and quickness. The network member profile comprises: General Information, Member Information, Request History, Member Preferences.

- The General Information part contains information about the member organisation, i.e. name of organisation, organization identifier in the system, date the organization was founded, and URL to the organization web page.

- Member Information is a set of tuples describing information about the member. Each tuple has the following properties:

o Member Name - the name of the member;

- Location - current geographical location of the member, it can be taken into account for estimating the speed and quality of request processing in a particular situation.

- Time - time zone of the member;

○ List of Languages - languages for contacting the member;

o Rights - knowledge area that the member can access;

- Group - the member can be part of a group, based on its capabilities;

o Phone Number, E-mail - contact information;

- Network Member Competence includes the following properties:

- Capabilities - types of operations that the member can implement;

- Capacities - capacity of the member;

- Prices - prices for implementing operations by the member;

- Velocity - velocity of implementation operation by this member.

- Member Sustainability describes sustainability properties of the member with regard to particular operations it can perform [1].

- Request History is a set of tuples. Each tuple possesses the following properties:

- Request - a request to the member;

o Context - is used to analyze the performance of the member (other members can see solutions generated in particular situations) and to identify detectable member preferences; 


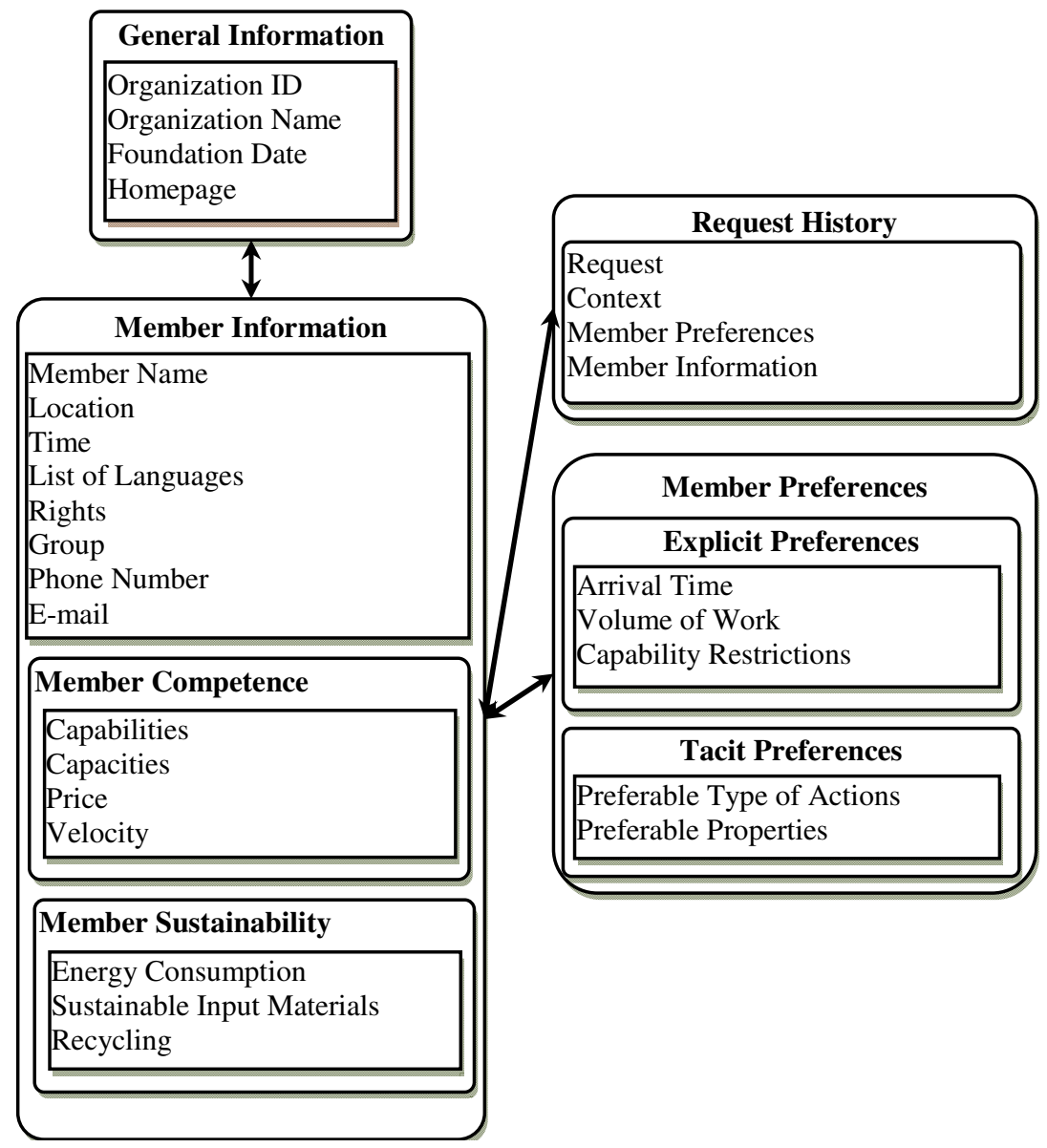

Fig. 3. Networked organisation member profile

- Member Preferences - stores member preferences at the moment of request initiation. They contain a snapshot of all the properties of the category "Network Member Preferences";

- Member Information - stores specific information about the member at the moment of request initiation. It contains a snapshot of all the properties of the category "Member Information".

- The Member Preferences part consists of Explicit Preferences and Tacit Preferences. Explicit Preferences describe member preferences that are manually introduced by a member. These preferences are used for choosing a member for a particular situation, and contain the member preference for arrival time, volume of work, and capability constraints. The latter stores several capabilities and logical restrictions from a list of all the capabilities for the domain. Tacit Preferences describe the automatically detectable member preferences. 


\section{Conclusions}

The paper describes an approach to decision support in flexible networked organisations. The approach is aimed at achieving a higher sustainability level due to maximisation of the flexibility and taking into account the sustainability properties of the organisation members. The members are proposed to be represented via Webservices. As a result the configuration of the flexible networked organisation can be replaced with that of services. Usage of context makes it possible to reduce the search space since it narrows the domain. Profiling is important for determining, which member is capable of carrying out a specified task with the given level of sustainability and, hence, can be chosen as a team member.

\section{Acknowledgments}

Some of the results are due to research carried out as a part of the project funded by grants \# 09-07-00436-a, 08-07-00264-a, and 09-07-00066-a of the Russian Foundation for Basic Research, and project \# 213 of the research program "Intelligent information technologies, mathematical modeling, system analysis and automation" of the Russian Academy of Sciences.

\section{References}

1. Seliger, G. (ed.): Sustainability in Manufacturing. Springer, Heidelberg (2007)

2. Allwood, J.: What is sustainable Manufacturing?

http: / / www. docstoc.com/docs/2319993/

What-is-Sustainable-Manufacturing

3. Smirnov, A., Pashkin, M., Chilov, N., Levashova, T., Krizhanovsky, A.: Fusion-based Intelligent Support for Logistics Management. Emerging Solutions for Future Manufacturing Systems. In: Camarinha-Matos, L.M. (ed.) Proceedings of IFIP TC 5 / WG 5.5 Sixth IFIP International Conference on Information Technology for Balanced Automation Systems in Manufacturing and Services (BASYS), pp. 209-216. Springer, Heidelberg (2004)

4. Dey, A.K.: Understanding and Using Context. Personal and Ubiquitous Computing J. 5(1), 4-7 (2001)

5. Uschold, M., Grüninger, M.: Ontologies: Principles, methods and applications. Knowledge Engineering Review 11(2), 93-155 (1996)

6. CADRC. KML Sandbox: An Experimentation Facility Based on SOA Principles. CADRD Currents, Fall, Collaborative Agent Design Research Center (CADRC), California Polytechnic State University, San Luis Obispo (2009) 